\title{
Roadmap to strengthen global mental health systems to tackle the impact of the COVID-19 pandemic
}

Pallab K. Maulik' ${ }^{1,23^{*}}$, Graham Thornicroft ${ }^{4}$ and Shekhar Saxena ${ }^{5,6}$

\begin{abstract}
Background: The COVID pandemic has been devastating for not only its direct impact on lives, physical health, socio-economic status of individuals, but also for its impact on mental health. Some individuals are affected psychologically more severely and will need additional care. However, the current health system is so fragmented and focused on caring for those infected that management of mental illness has been neglected. An integrated approach is needed to strengthen the health system, service providers and research to not only manage the current mental health problems related to COVID but develop robust strategies to overcome more long-term impact of the pandemic. A series of recommendations are outlined in this paper to help policy makers, service providers and other stakeholders, and research and research funders to strengthen existing mental health systems, develop new ones, and at the same time advance research to mitigate the mental health impact of COVID19. The recommendations refer to low, middle and high resource settings as capabilities vary greatly between countries and within countries.

Discussion: The recommendations for policy makers are focused on strengthening leadership and governance, finance mechanisms, and developing programme and policies that especially include the most vulnerable populations. Service provision should focus on accessible and equitable evidence-based community care models commensurate with the existing mental health capacity to deliver care, train existing primary care staff to cater to increased mental health needs, implement prevention and promotion programmes tailored to local needs, and support civil societies and employers to address the increased burden of mental illness. Researchers and research funders should focus on research to develop robust information systems that can be enhanced further by linking with other data sources to run predictive models using artificial intelligence, understand neurobiological mechanisms and community-based interventions to address the pandemic driven mental health problems in an integrated manner and use innovative digital solutions.
\end{abstract}

Conclusion: Urgent action is needed to strengthen mental health system in all settings. The recommendations outlined can be used as a guide to develop these further or identify new ones in relation to local needs.

Keywords: Mental health systems, COVID 19, Mental health services, Research, Mental health resources, Policy making

*Correspondence: pmaulik@georgeinstitute.org.in

${ }^{1}$ George Institute for Global Health, 311-312 Elegance Tower, Jasola, New Delhi 110025, India

Full list of author information is available at the end of the article

\section{Background}

The COVID-19 pandemic is not only affecting communities directly, but through its socio-economic consequences is also affecting the lives of many millions of families and individuals. The direct health impact, economic impact, and disruption of the social and 
community structures across the globe is potentiating a major international mental health crisis [1-4]. The mental health impacts of COVID-19 can be varied and severe and have been outlined recently [5]. The effect of this stress can vary from mild symptoms related to physiological or psychological functions such as sleep disturbance or low mood, mild stress for short periods of time that do not need any specific treatment and resolve when the primary stressors such as job loss or illness in family or poor social support are taken care off, to the more severe syndromal mental disorder which may need formal treatment from a mental health professional [6].

Anxiety, depression, increased alcohol and substance use, irritability, anger, insomnia and increased risk of suicide have been reported, as have been risk factors for mental disorders such as loneliness, domestic violence, physical violence. Individuals with existing mental disorders such as alcohol and substance use, cognitive impairment and dementia, childhood psychiatric disorders and adults needing long term follow up have been particularly affected due to lack of continued psychiatric care services and fragmentation of the existing health systems to provide adequate care. In addition, the direct impact of COVID 19 on mental illness of those infected or health workers involved in care of those infected is also significant, and is often precipitated due to increased stigma, social isolation and quarantine [5]. All this is even more complicated due to the socioeconomic impact of the pandemic on the lives of the poor and most disadvantaged communities such as homeless and migrant workers. The overall mental health impact of the pandemic is not transient but likely to continue for a long period even after the pandemic ends, as is evident from prior research on such severe epidemics $[7,8]$. Researchers have highlighted the need for focussed research that should be funded related to the impact of COVID-19 [9].

\section{The current challenge}

Most mental health systems across the world have been woefully inadequately funded, planned, organised and delivered given the major global burden of mental disorders [10]. The CODID-19 pandemic has added even greater challenges. With shrinking economies, policy makers will have to rebalance prioritizing mental health services against other health service investments. The ability to react and take appropriate decisions will depend on the existing resources and infrastructure. These decisions will then need to be matched up against the impact of the pandemic-not only on mental health, but to the overall health of the country, as well as the socioeconomic determinants. Thus, it becomes important to have a better understanding of what steps can be taken in such scenarios to make most efficient use of the limited resources. At the same time new research should align with the changing paradigm of mental health care delivery which may have to rely on use of digital solutions [11], identify risk factors that are particularly relevant to precipitating mental disorders in the face of this pandemic, and develop and implement scalable interventions to mitigate the impact of the infection on mental health across different communities and different settings.

In this context, the aim of this paper is to outline a roadmap to guide countries to strengthen mental health systems to tackle the increasing burden of mental disorders. Using both World Health Organization's Mental Health Action Plan 2013-2020 [12] and the WHO health systems strengthening framework [13], we propose a set of recommendations from the perspectives of policy makers, service providers and research funders, organised into low-, middle- and high-resource scenarios. While the recommendations encompass systematic and structural actions that are relevant to building a strong mental health system per se and is essential to the current pandemic as in any other crisis, embedded within them are some more specific aspects that are particularly relevant to the COVID crisis, and these have been indicated separately. The eventual objective is to "build back better" [14].

\section{Recommendations to overcome the challenge}

Table 1 shows recommendations for policy makers in areas of leadership and governance, finance, policies and programmes that include long term care and needs of vulnerable populations.

Table 2 outlines recommendations for service providers and other stakeholders involved in care of those with mental health problems. It focuses on providing equitable and accessible community-based mental health services and clinic-based services for those needing such care, build capacity by training primary care health workers to provide community-based services, implement community-based mental health prevention and promotion programmes, strengthen civil societies to support the government mental health service provision, and support programmes and policies specifically to manage workplace related stress which will be a major issue given the economic woes and changing paradigms of limited workforce or working from home.

Table 3 outlines recommendations for researchers and research funders to align research to strengthen information systems, gather more epidemiological data and conduct robust interdisciplinary interventions that are scalable, use innovative designs and leverage technology to develop some interventions to facilitate service delivery and improve supply chain of psychotropic medications at primary care levels and leverage the power of social media to deliver interventions. Technology 


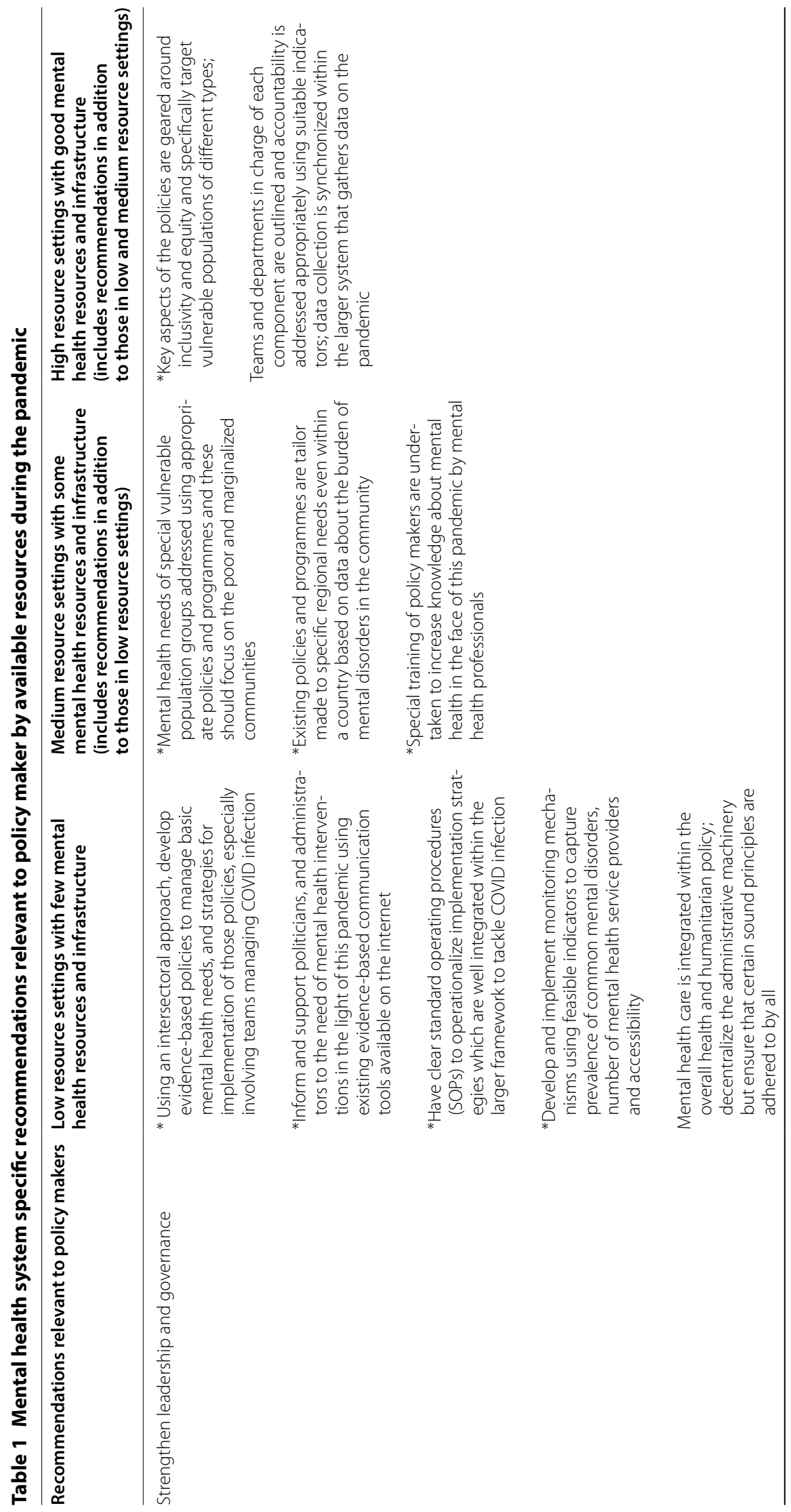




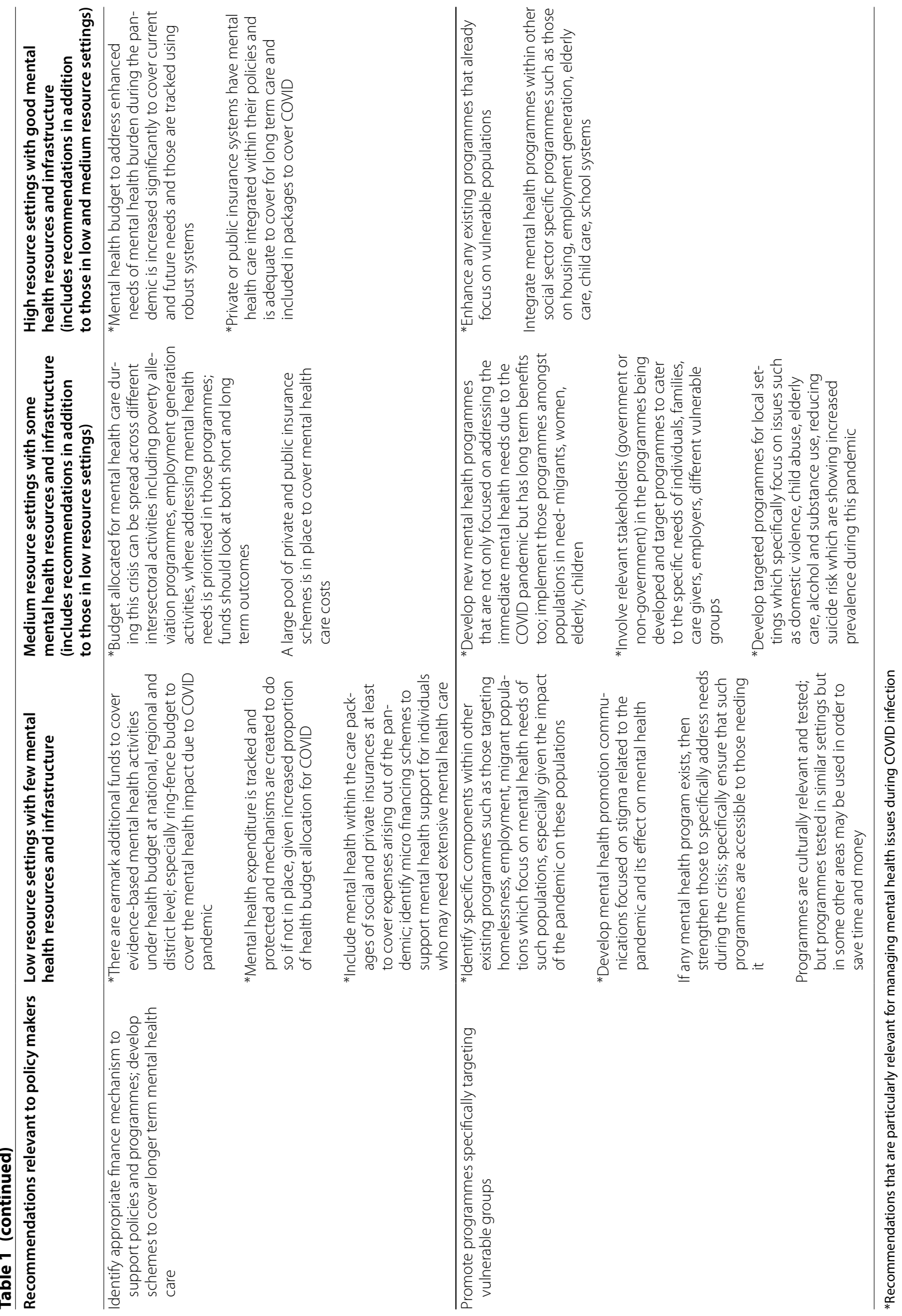




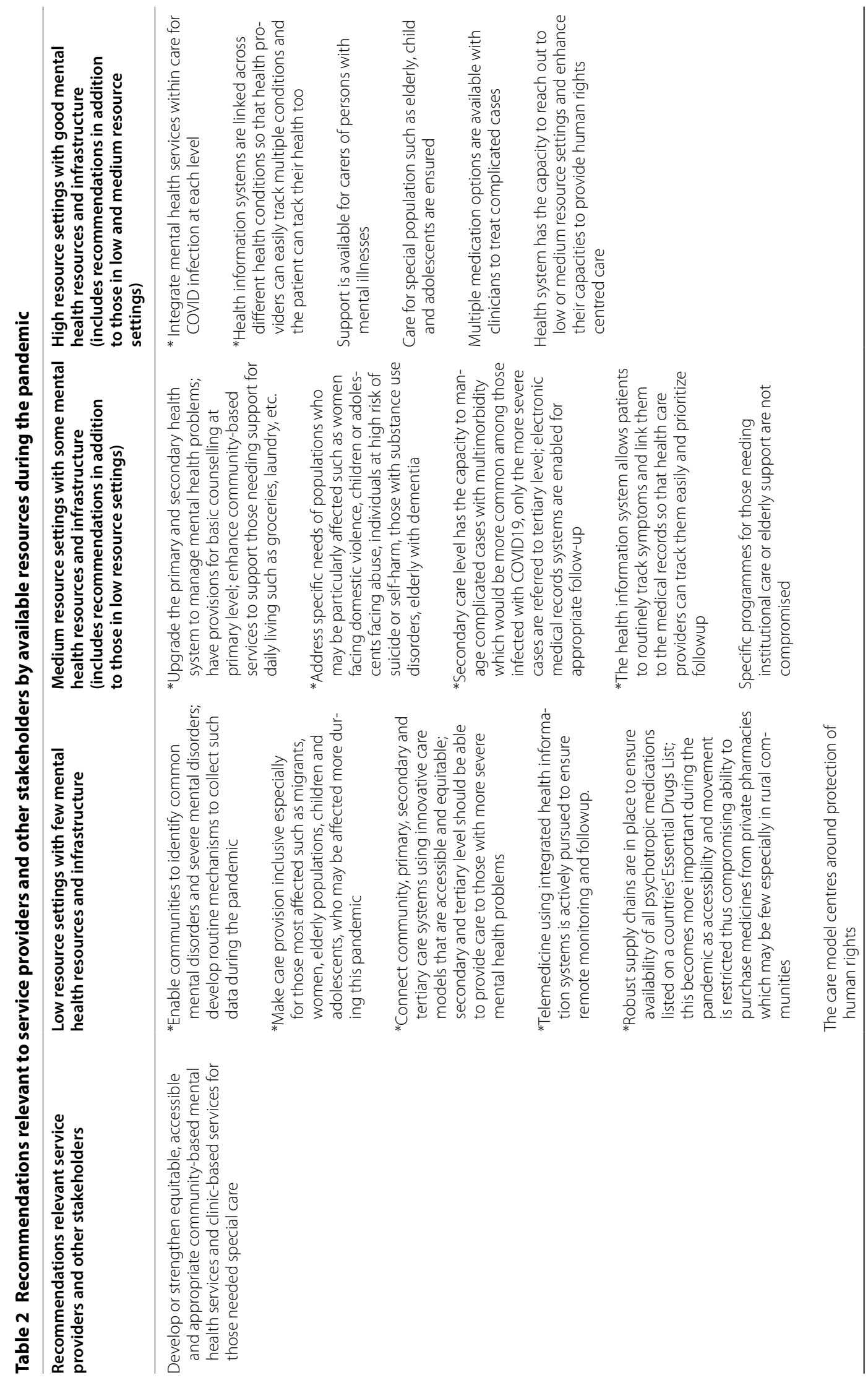




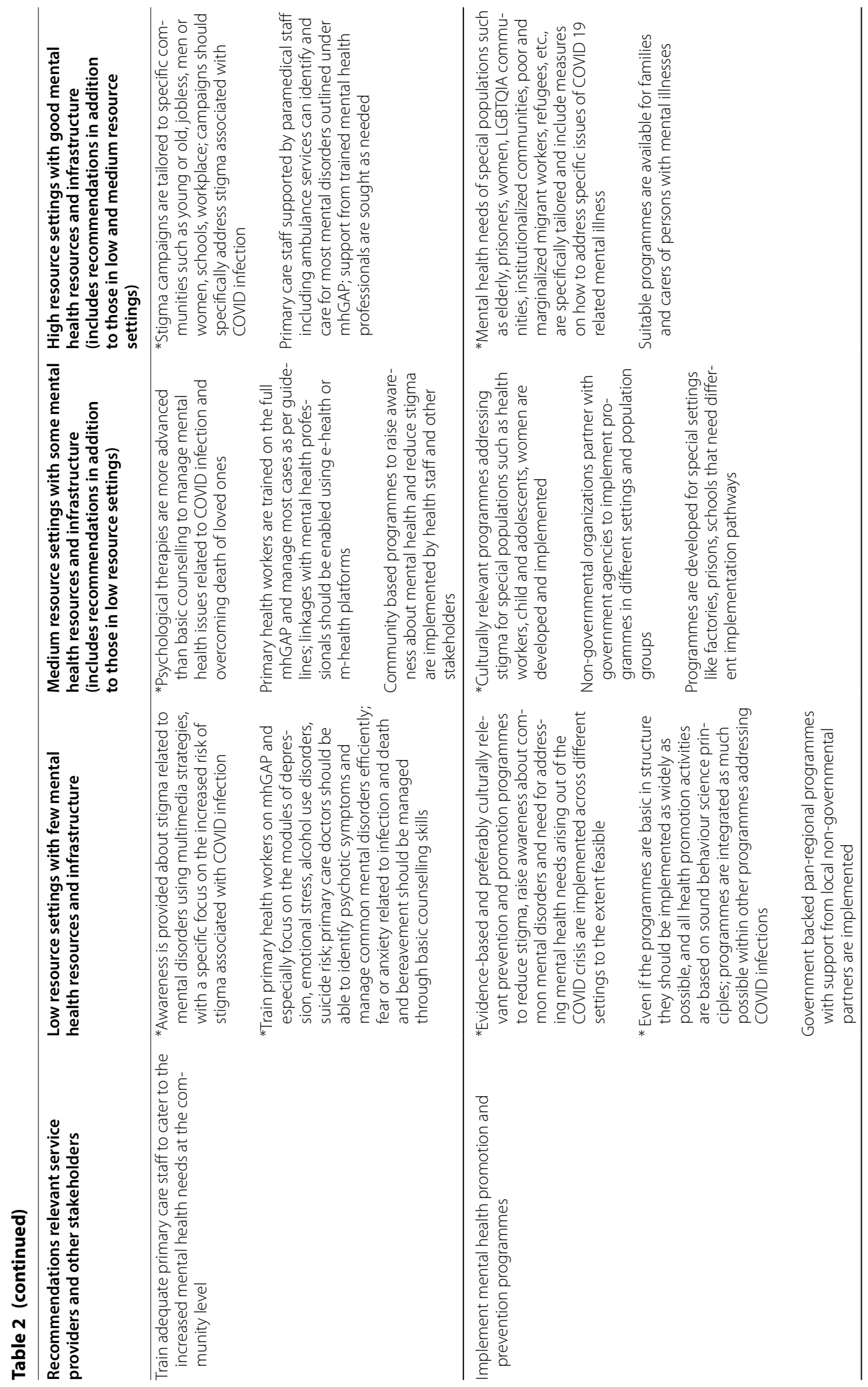




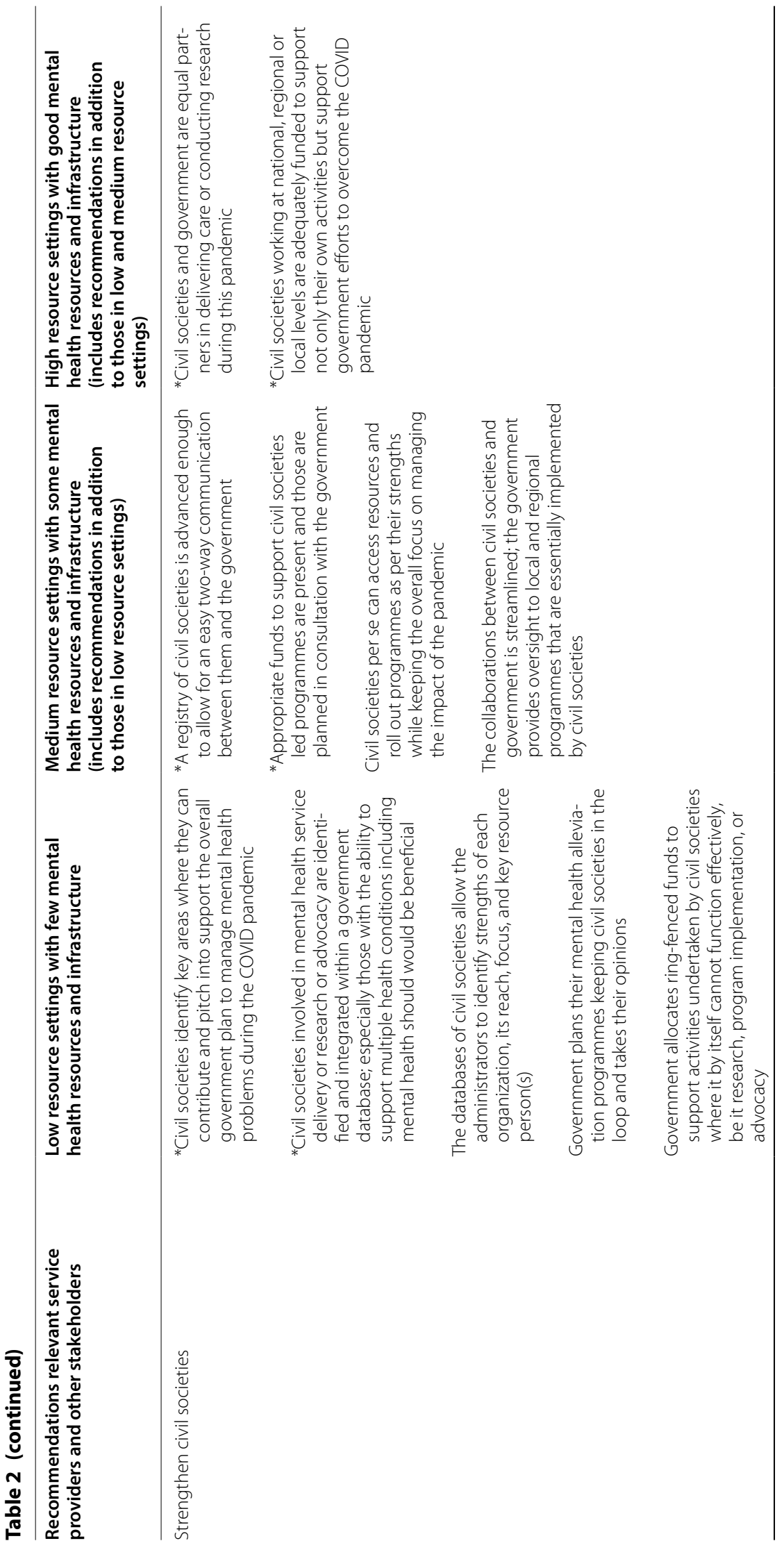




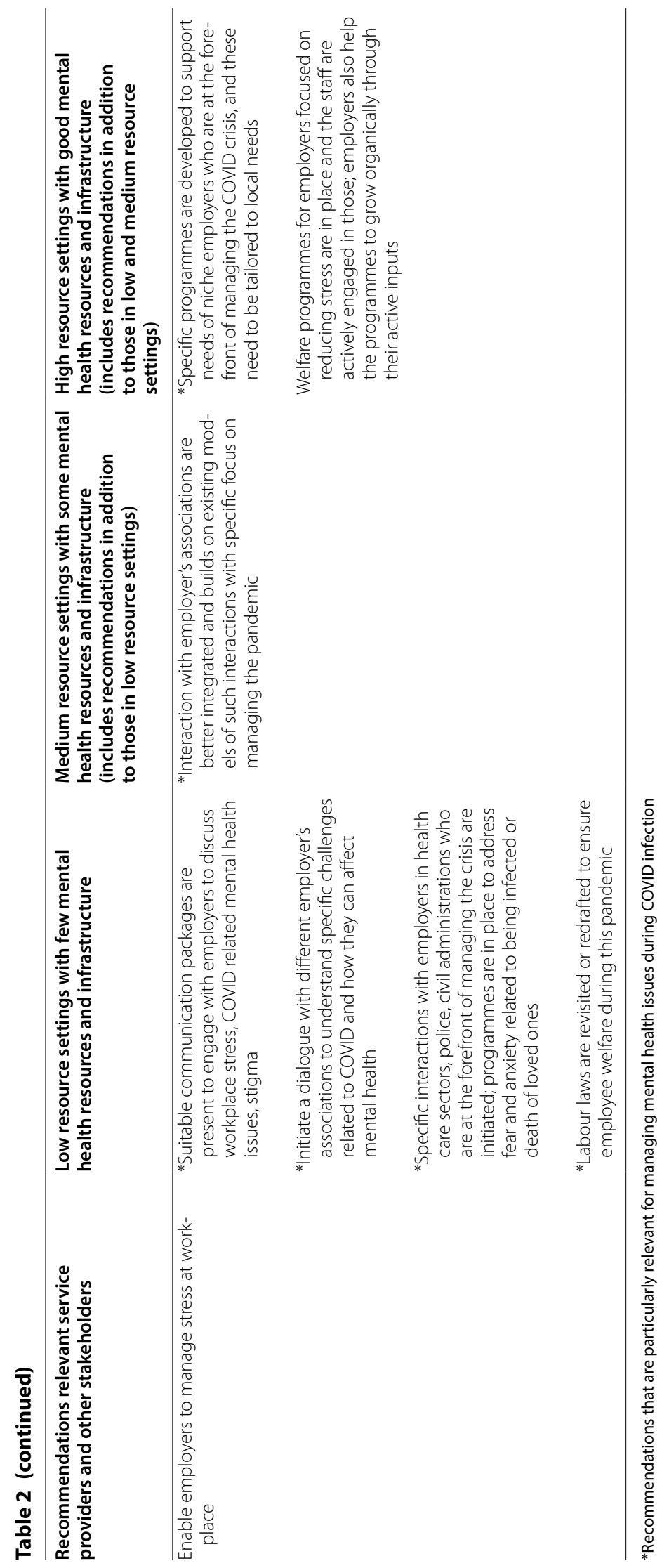




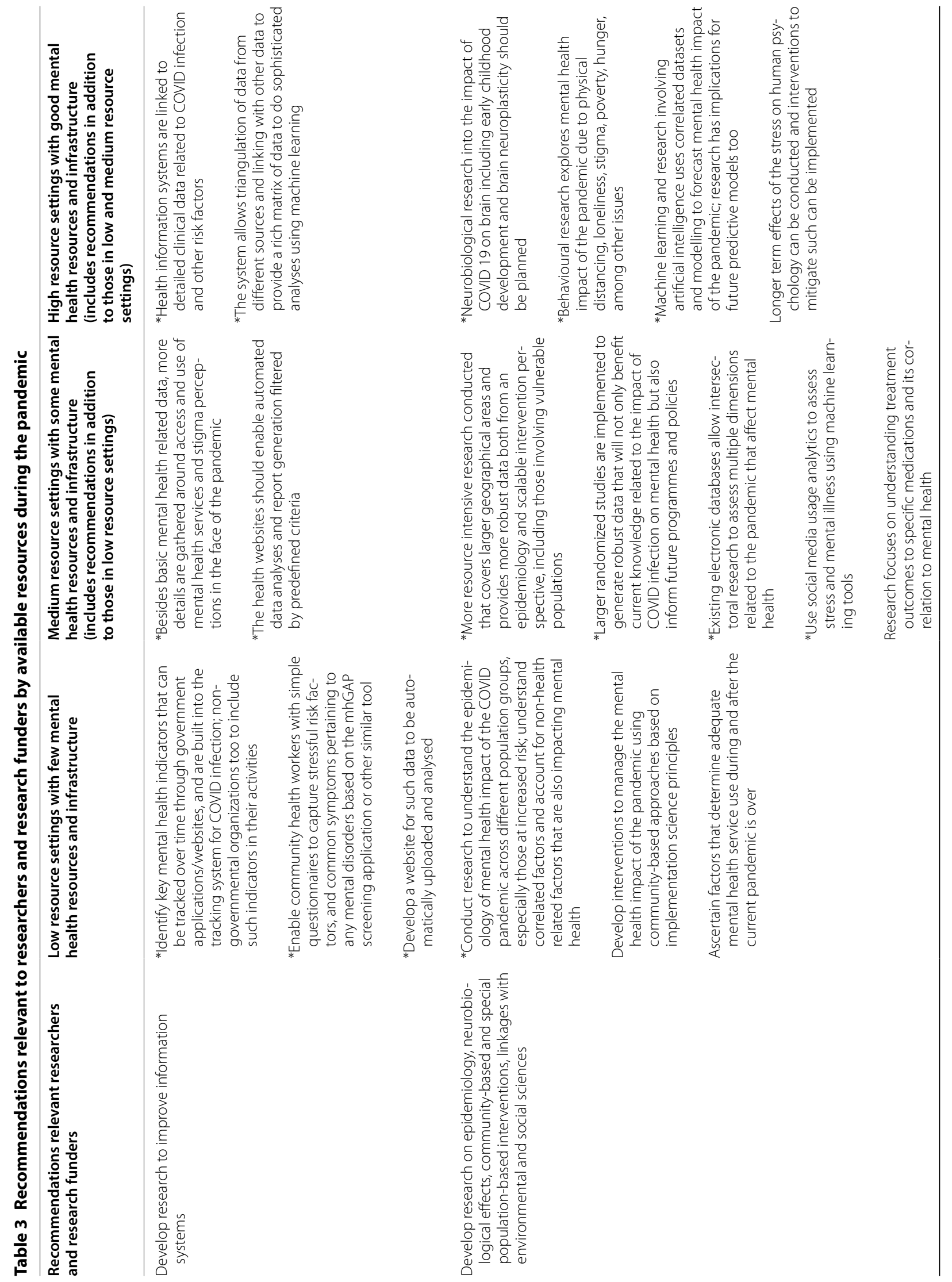




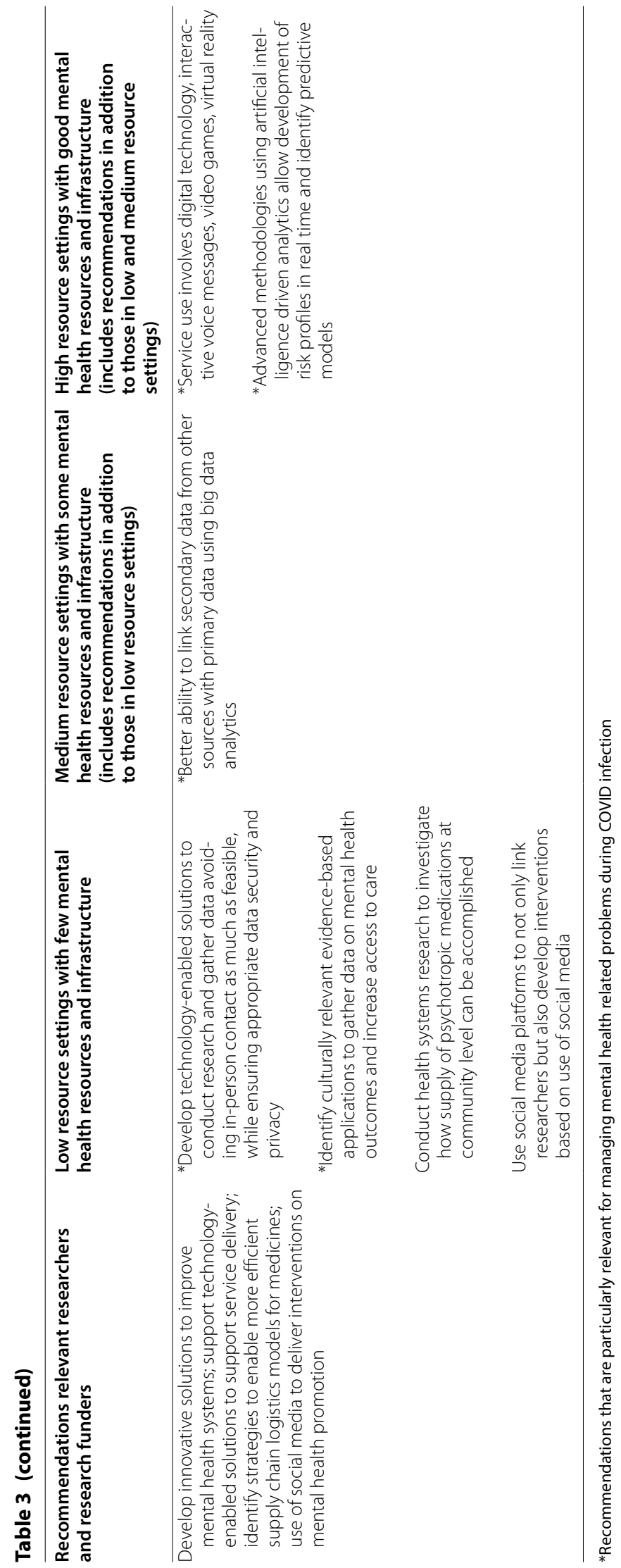


led solutions need to be ramped up especially in these conditions where in-person data collection is limited considerably.

\section{Discussion}

Mental health is one of the most neglected areas of health. The COVID 19 pandemic and any similar challenges in future, should be tackled along the lines of a humanitarian emergency [15]. Even during more normal times, addressing mental health needs as part of the Sustainable Development Goals has been a major challenge [6]. The COVID crisis has led to a fragmentation of existing health systems across the globe, which will have a profoundly negative and cascading effect on mental health not only in coming months, but for some years, and this has been identified even at the United Nations [2]. Not only will COVID 19 lead to a surge in mental health needs in the community $[5,7]$, but the way it has crippled the health systems globally to address the need of any other health problem, it is likely to have a devastating effect on the longer term needs of people who need care for mental illnesses [5].

\section{Strengthening existing mental health systems to tackle the pandemic}

It becomes necessary to identify strategies to strengthen health systems to overcome these challenges. The best way to tackle mental health impact is to not limit it to overcoming the immediate mental health crisis, but to embed its management within the larger health system that can impact the lives of individuals globally or across large regions. In this paper we have focused on low, middle and high resource settings and indicated how they can re-orient their health systems, service provision and research according to the need and available resources. This approach applies as much to countries as it does to regions or health administrative units within countries, given the very large disparities in needs and resources that are common in countries worldwide. We present the recommendations in Tables 1, 2 and 3 not as separate and unrelated proposals, but as part of an overall integrated approach to health system strengthening, which should be adapted to specific local needs and modified in relation to available resources.

\section{Recommendations for policy makers}

Policy makers will play a major role in providing leadership to any programmes and policies that they develop and implement. It is therefore imperative that they are both educated about the mental health needs during this crisis and supported by academicians and mental health professionals to develop robust policies and programmes to address the increased burden of mental health. While there will be a requirement to address some immediate mental health needs and provide psychosocial support in line with the IASC guidelines [15], they should plan on developing more robust policies and programmes to build a system that is more holistic, encompasses intersectoral collaborations, protects the rights of the individuals, has deliverables that are based on evidence, and is able to deliver care over a long time.

These policies and programmes should be supported by adequate funding and tap into existing private and government sources. Insurance mechanisms should ensure that adequate financial support is available for individuals to seek mental health care as per need. This may need a paradigm shift in the way the insurance system is organized as most often mental disorders are excluded from their remit. In United States of America, telehealth parity has been introduced in many other states post the COVID crisis to ensure providers get same payment for teleconsultations as in-person consultations, thus enabling service delivery [16]. Telepsychiatry has also resulted in expanding home-based care for conditions like substance use disorders in the United State, which earlier were only available if comorbid physical disorders were present. Policy makers should support development of teleconsultations and robust electronic health records systems to enable remote care delivery.

The mental health budget allocation should reflect the change in the burden due to the crisis and the government should be open to exploring innovative ways to build in mental health related budget into the relevant sectors, for example, addressing job security, providing affordable homes for migrant workers, building shelters for women or children facing abuse, enhancing care for the elderly and those with dementia, could help in reducing the burden considerably. Strategies should be locally relevant and keep needs of vulnerable populations, inclusivity, stigma reduction, and rights-based approaches at the core of their principles [12].

\section{Recommendations for service providers and other stakeholders}

The key elements that service providers should keep in mind are to develop a model that is community-based and involves training and upskilling of primary health workers and non-mental health professionals to both identify and deliver basic mental health care based on principles laid down by existing guidelines [12], and drawing on basic tenets and the detailed guidance of the mhGAP programme of the World Health Organization. Psychological therapies can be tailored to the level of skilled resources available.

The level of specialized care provided should be informed by local factors and available resources. Some 
of those are number of mental health trained staff and their skills level, types of mental health facilities available, for example primary, secondary or tertiary care, budgets available to support services, availability of communitybased support services to cater to specific needs of individuals with significant disabilities, support services for families and caregivers, role of multi-sectoral agencies to support mental health care such as employment agencies, housing, elderly welfare, child welfare services, education. Services provided should be locally tested and culturally relevant. Needs of vulnerable populations should be specially kept in mind. The services should be both accessible and equitable, and one key strategy to ensure that in times of physical distancing could be increased use of technology enabled services such as e-health, m-health, telemedicine $[11,17]$. This should encompass screening, service delivery, training of health workers and monitoring.

A key aspect is to maintain physical distancing while ensuring continuity of care. To do so telemedicine services and linking of patient and provider data on health information systems that enables tracking of a patient's health remotely is necessary. The system should allow both the patient and health providers to interact with each other either through video chats or dedicated phone lines and be interactive enough to allow the patient to upload their progress, treatment adherence and complications online and the provider can respond to those in real time. Reports from Italy, underline how mental health services were prioritized in the face of the COVID pandemic by identifying essential mental health services, providing medications to those with substance use disorders, enabling teleconsultations [18, 19]. Even in low resource settings such as In India, teleconsultation for mental health issues is being regularly provided by many tertiary care centres, though there is a lot of scope for improvement. Civil societies have also set up teleconsultation to care for emergency situations [20]. In China, there were more specific challenges as being the first country to face the pandemic, there were no prior experiences to follow, but restructuring of service at different levels and delivering a mix of online and offline services were identified as critical for ensuring continuity of care, but new ethical challenges related to teleconsultations and practical problems related to implementation of new strategies had to be overcome [21].

Availability of psychotropic medicines should be facilitated by ensuring that the supply-chain is maintained, and governments need to invest for that specifically in low and middle resource settings. Civil societies should be encouraged to collaborate with government agencies and work in both strategizing and service delivery and the government should allocate ring-fenced funds for such activities. Labour organizations and employers should be adequately trained to identify specific mental health needs of individuals in this pandemic, but also encouraged to revisit their policies to ensure that their laws are employer friendly but also allowing for industry growth. Addressing the mental health needs of employees is critical even in normal times [22] and during this added challenge it may be a major factor to alleviate the burden as employees and employers both grapple with new situations of working from home, restricted office attendance, staff layoff, reduced productivity, and reduced remunerations.

\section{Recommendations for researchers and research funders}

The focus of research and the level of sophistication of such will vary across low, medium and high resource settings. Even within a high-income country there may be a need to understand how to deliver basic services or ascertain prevalence or incidence of mental disorders in some regions with lower resources. In order to capture the true burden of COVID 19 on mental health, it is vital that information systems to gather such data is strengthened across all settings. It is important to create a system where data from multiple sources can be linked to build an aggregate database involving both clinical and social determinants. An initiative on this, Countdown Global Mental Health 2030 is already underway [23]. Research exploring neurobiological correlates, behavioural concepts that determine how stigma and discrimination plays a role in help seeking in COVID affected individuals, effect of socioeconomic policies on mental health, mental health effects on different populations by age groups, gender, migrant and labourer communities, homeless, health workers, etc., are all relevant areas of further investigation $[9,24,25]$. Research should also explore newer strategies using machine learning and artificial intelligence to build predictive models to inform risk profiles for future pandemics and determine possible phenotypes that could allow service providers to modulate care and overall outcomes. The role of artificial intelligence, digital tools to collect real-time data, combining online and off-line data with in-person data needs to be enabled to enrich research data to support better care models [26].

\section{Conclusion}

We believe that urgent action is needed to strengthen mental health system in all settings in view of enhanced need for mental health care and decreased access during and beyond the COVID-19 pandemic. The roadmap draws upon key sources and accumulated knowledge of mental health systems globally to provide a perspective on practical steps to strengthen mental health systems across the world. The strategies outlined here can be used as a guide to develop these further or identify new ones that are more applicable to local settings. Taking no 
action in the face of increasing threats to mental health of populations is not an option in the COVID era. The roadmap that we recommend here is intended to be used as a guide by policy makers, service providers and other stakeholders, researchers and research funders to develop strategies to actively improve mental health in relation to COVID 19 following the principle of building back better [14] and deliberations of the National Academy of Sciences where suggestions were made to have person centred care, shared decision making and patient and family engagement [27], and to make mental health an integral part of the management of COVID 19 [28].

\section{Acknowledgements}

PKM is supported through NHMRC/GACD grant (SMART Mental Health APP1143911). GT is supported by the National Institute for Health Research (NIHR) Applied Research Collaboration South London at King's College London NHS Foundation Trust, and by the NIHR Asset Global Health Unit award. The views expressed are those of the author(s) and not necessarily those of the NHS, the NIHR or the Department of Health and Social Care. GT also receives support from the National Institute of Mental Health of the National Institutes of Health under award number R01MH100470 (Cobalt study). GT is supported by the UK Medical Research Council in relation the Emilia (MR/ S001255/1) and Indigo Partnership (MR/R023697/1) awards.

\section{Authors' contribution}

PKM led the development of the manuscript and wrote the first draft and all subsequent drafts. GT and SS provided critical comments to the first draft and each subsequent draft. All authors read and approved the final manuscript.

\section{Competing interests}

The authors declare that they have no competing interests.

\section{Author details}

${ }^{1}$ George Institute for Global Health, 311-312 Elegance Tower, Jasola, New Delhi 110025, India. ${ }^{2}$ University of New South Wales, Sydney, Australia.

${ }^{3}$ Prasanna School of Public Health, Manipal, India. ${ }^{4}$ Centre for Global Mental Health and Centre for Implementation Science, Institute of Psychiatry, Psychology and Neuroscience, King's College London, London, UK. ${ }^{5}$ Global Health and Population Harvard T H Chan School of Public Health, Boston, USA.

${ }^{6}$ George Institute for Global Health, Sydney, Australia.

Received: 19 June 2020 Accepted: 23 July 2020

Published online: 29 July 2020

\section{References}

1. Auerbach J, Miller BF. COVID-19 exposes the cracks in our already fragile mental health system. Am J Public Health. 2020. https://doi.org/10.2105/ AJPH.2020.305699.

2. United Nations. Policy brief: COVID 19 and the need for action on mental heath. New York: United Nations; 2020.

3. Editorial. Mental health and COVID-19: change the conversation. Lancet Psychiatry. 2020;7(6):463.

4. Adhanom Ghebreyesus T. Addressing mental health needs: an integral part of COVID-19 response. World Psychiatry. 2020;19(2):129-30.

5. Vigo D, Patten S, Pajer K, Krausz M, Taylor S, Rush B, et al. Mental health of communities during the COVID-19 pandemic. Can J Psychiatry. 2020. https://doi.org/10.1177/0706743720926676.

6. Patel V, Saxena S, Lund C, Thornicroft G, Baingana F, Bolton P, et al. The Lancet Commission on global mental health and sustainable development. Lancet. 2018;392(10157):1553-98.

7. Hong X, Currier GW, Zhao X, Jiang Y, Zhou W, Wei J. Posttraumatic stress disorder in convalescent severe acute respiratory syndrome patients: a 4-year follow-up study. Gen Hosp Psychiatry. 2009;31 (6):546-54.
8. Rogers JP, Chesney E, Oliver D, Pollak TA, McGuire P, Fusar-Poli P, et al. Psychiatric and neuropsychiatric presentations associated with severe coronavirus infections: a systematic review and meta-analysis with comparison to the COVID-19 pandemic. Lancet Psychiatry. 2020. https://doi. org/10.1016/S2215-0366(20)30203-0.

9. Holmes EA, O'Connor RC, Perry VH, Tracey I, Wessely S, Arseneault L, Ballard C, Christensen H, Cohen Silver R, Everall I, et al. Multidisciplinary research priorities for the COVID-19 pandemic: a call for action for mental health science. Lancet Psychiatry. 2020;7(6):547-60.

10. World Health Organization. Mental Health Atlas 2017. Geneva: World Health Organization; 2018.

11. Mahmood S, Hasan K, Colder Carras M, Labrique A. Global preparedness against COVID-19: we must leverage the power of digital health. JMIR Public Health Surveill. 2020;6(2):e18980.

12. World Health Organization. Mental health action plan 2013-20. Geneva: World Health Organization; 2013.

13. World Health Organization. Everybody business: strengthening health systems to improve health outcomes: WHO's framework for action. Geneva: World Health Organization; 2007.

14. World Health Organization. Building back better: sustainable mental health care after emergencies. Geneva: World Health Organization; 2013.

15. Committee IASC. IASC Guidelines on Mental Health and Psychosocial Support in Emergency Settings. Geneva: IASC; 2007.

16. Warren JC, Smalley KB. Using telehealth to meet mental health needs during the COVID-19 crisis. To the Point (blog), Commonwealth Fund, June 18, 2020. https://www.commonwealthfund.org/blog/2020/using -telehealth-meet-mental-health-needs-during-covid-19-crisis.

17. Drew DA, Nguyen LH, Steves CJ, Menni C, Freydin M, Varsavsky T, et al. Rapid implementation of mobile technology for real-time epidemiology of COVID-19. Science. 2020. https://doi.org/10.1126/science.abc0473.

18. D'Agostino A, Demartini B, Cavallotti S, Gambini O. Mental health services in Italy during the COVID-19 outbreak. Lancet Psychiatry. 2020;7(5):385-7.

19. Percudani M, Corradin M, Moreno M, Indelicato A, Vita A. Mental Health Services in Lombardy during COVID-19 outbreak. Psychiatry Res. 2020;288:112980.

20. iCALL Psychosocial Helpline. Tata Institute of Social Sciences; 2020. http:// icallhelpline.org/covid-19/.

21. Chen S, Li F, Lin C, Han Y, Nie X, Portnoy RN, Qiao Z. Challenges and recommendations for mental health providers during the COVID-19 pandemic: the experience of China's First University-based mental health team. Global Health. 2020;16(1):59.

22. Maulik PK. Workplace stress: a neglected aspect of mental health wellbeing. Indian J Med Res. 2017;146(4):441-4.

23. Saxena S, Kestel D, Sunkel C, London E, Horton R, Patel V, et al. Countdown global mental health 2030. Lancet. 2019;393(10174):858-9.

24. Fegert JM, Vitiello B, Plener PL, Clemens V. Challenges and burden of the Coronavirus 2019 (COVID-19) pandemic for child and adolescent mental health: a narrative review to highlight clinical and research needs in the acute phase and the long return to normality. Child Adolesc Psychiatry Ment Health. 2020;14:20.

25. Vahia IV, Blazer DG, Smith GS, Karp JF, Steffens DC, Forester BP et al. COVID-19, mental health and aging: a need for new knowledge to bridge science and service. Am J Geriatr Psychiatry. 2020;S1064-7481(20):30271-2.

26. Balcombe L, de Leo D. Commentary: an integrated blueprint for digital mental health services amidst COVID-19. JMIR Ment Health. 2020. https:// doi.org/10.2196/21718.

27. National Academies of Sciences, Engineering, and Medicine 2020. Key policy challenges and opportunities to improve care for people with mental health and substance use disorders: proceedings of a workshop. Washington, DC: The National Academies Press. https://doi.org/10.17226 /25690.

28. Campion J, Javed A, Sartorius N, Marmot M. Addressing the public mental health challenge of COVID-19. Lancet Psychiatry. 2020. https://doi. org/10.1016/S2215-0366(20)30240-6.

\section{Publisher's Note}

Springer Nature remains neutral with regard to jurisdictional claims in published maps and institutional affiliations. 